\title{
Interactive large group teaching for final-year medical students; a method to prepare students for the Prescribing Safety Assessment and beyond
}

\author{
Authors: Rebecca C Stout and Narin Shareef
}

\begin{abstract}
Aims
Prescribing is a key skill for medical students to learn. All finalyear students must now undertake the Prescribing Safety Assessment (PSA) prior to being able to graduate and begin work as a foundation doctor within the UK. The aim of our study was to create an interactive and practical prescribing skills teaching session which would equip students with the tools they need to prescribe both for their PSA and for their future practice as junior doctors.
\end{abstract}

\section{Methods \\ The teaching session was designed to be assessment-centred, covering all eight domains that are assessed within the PSA as a way of ensuring students remained engaged. All students were provided with a drug kardex on which they could practice prescribing. Case studies were worked through, with 'breakout' activities which enabled students to practice prescribing on their drug kardexes and to answer key questions about prescribing. Students were provided with the British National Formulary (BNF), and encouraged to download the $B N F$ application onto their phones before the teaching session to aid prescribing and make it as 'near to life' as possible. Students were given the opportunity to discuss the case in pairs, and to have a few minutes to decide what to prescribe, before feeding back and checking whether their answer was correct. At the end of the session, students were asked to complete feedback about how they had found the teaching session.}

\section{Results}

Students particularly found that the large group, interactive teaching sessions were useful in adding to their current prescribing knowledge and skills, with $83 \%$ reporting that the teaching had added to this. Ninety per cent felt more confidently prepared for their PSA and $70 \%$ felt more prepared in beginning prescribing as foundation-year doctors after the session. In our qualitative feedback replies students found it useful to go through questions in the format of the exam, to practice prescribing on real drug kardexes and to go through case-based scenarios instead of receiving more classical didactic based lectures. Areas for improvement included that students would have liked to have gone over how to use a $B N F$, and that it would be useful to have such a session prior to going into the clinical area on placements.

\section{Conclusion}

We have demonstrated that even in large groups, interactive teaching can be used to deliver prescribing skills sessions. Students found this particularly useful when using drug kardexes on which to prescribe and when teaching was linked to their future examinations. This is a method which could be adopted by universities throughout the UK as a method of delivering this type of teaching to large groups of students at the same time.

\section{Conflict of interest statement}

Students were charged $£ 10$ to attend this teaching which covered printing costs. All subsequent profits were donated to the Hand in Hand for Syria charity once these costs had been covered. 